\title{
Effect of shared decision-making on therapeutic alliance in addiction health care
}

\author{
EAG Joosten ${ }^{1,2}$ \\ $\mathrm{GH}$ de Weert ${ }^{3}$ \\ T Sensky ${ }^{4}$ \\ CPF van der Staak ${ }^{5}$ \\ CAJ de Jong ${ }^{1,2}$
}

'Novadic-Kentron, Network for Addiction Treatment Services, Vught, the Netherlands; ${ }^{2}$ Nijmegen Institute for Scientist-Practitioners in Addiction (NISPA), Nijmegen, the Netherlands; ${ }^{3}$ Julius Center for Health Sciences and Primary Health Care, UMC Utrecht, Utrecht, the Netherlands; ${ }^{4}$ Department of Psychological Medicine, Imperial College London, London, United Kingdom; ${ }^{5}$ Academic Centre for Social Sciences, Radboud University Nijmegen, Nijmegen, the Netherlands

Correspondence: Evelien Joosten Nijmegen Institute for ScientistPractitioners in Addiction, PO Box 9104 , $6500 \mathrm{HE}$ Nijmegen, the Netherlands

Tel $+312436 I I I 50$

Fax +3I 2436 III52

Email evelien.joosten@novadic-kentron.nl (EAG Joosten)
Background: In recent decades, shared decision-making (SDM) models have been developed to increase patient involvement in treatment decisions. The purpose of this study was to examine the effect of a shared decision-making intervention (SDMI) for substance-dependent patients on patients' and clinicians' perceptions of therapeutic alliance.

Methods: Clinicians were randomly assigned to SDMI or usual procedures to reach a treatment agreement. SDMI is a structured, manualized, 5-session procedure to facilitate treatment agreement and consists of five standardized sessions.

Results: Patients' perceptions of the therapeutic alliance were very favorable at start of treatment, and no differences were found between intervention groups. Clinicians' scores on perceived helpfulness and on the overall therapeutic alliance were higher in the SDMI group than in the controls, after 8 weeks of treatment and at the end of treatment.

Conclusion: The present study has shown that a specific intervention to enhance shared decision-making results in favorable changes in clinicians' perceptions of the therapeutic alliance.

Keywords: therapeutic alliance, helping alliance, shared decision-making, addiction, substance-dependence

\section{Introduction}

Studies in the recent decades have demonstrated that the quality of the therapeutic relationship is a significant predictor of treatment adherence and outcome in addiction health care (Meier et al 2005). The collaborative aspects of the therapeutic relationship are generally described as therapeutic alliance (Hougaard 1994). Therapeutic alliance is not equal to therapeutic relationship, although the relationship may influence alliance. It is formed in the complex interaction between the patient and the clinician (Baldwin et al 2007). Therapeutic alliance has been defined in a number of ways but most definitions identify therapeutic alliance as the mutual collaboration between clinician and patient, which includes emotional bonding, a sense of warmth and openness, and shared expectations of both tasks and goals of therapy (Connors et al 1997). Therapeutic alliance related terms are working alliance and helping alliance. Working alliance emphasizes patient's ability to work purposively in the treatment situation (Greenson, 1965). Helping alliance concerns patient's experience of treatment or relationship with the clinician as helpful or potentially helpful (Luborsk 1976).

One aspect of this definition is the patient-clinician agreement on tasks and goals. This aspect is strongly related to shared decision-making (SDM) (Fische 2006). In recent years, the patient-clinician relationship has become more of a partnership or a collaboration (Charles et al 1999). Clinicians now adopt more of a "we" rather than a "me" approach to treatment (Fuertes et al 2007). SDM is a new and important aspect in such collaboration. SDM is defined as an approach in which clinician and patient go through all phases of the decision-making process together and in which they 
share the preference for treatment and reach an agreement on treatment choice (Charles et al 1999). Legislation has been passed in several countries aimed at strengthening the influence of patients (Crawford et al 2003). In the Netherlands, patient involvement in decision-making about treatment has been formally prescribed in a specific law (WGBO), and the principle of identifying patient's view is increasingly presented in national guidelines for health care professionals (Van der Weijden et al 2007).

In the treatment of substance abuse there is, as in somatic care, increasing emphasis on patient autonomy and active involvement in decision-making (Robinson and Thomson 2001). De Jong-Verhagen and De Jong (2001) recommended the use of a structured procedure to systematically list and rank patients' goals and expectations, preferably at different times during treatment. This procedure leads to a dialogue between clinician and patient regarding treatment goals and expectations, resulting in a treatment agreement which may be adapted during treatment. Discussing with patients about choices, especially about the goal of treatment, is expected to lead to better treatment outcome (Sanchez-Craig 1990). This dialogue between patient and clinician is also expected to lead to improvement in the therapeutic alliance (Hamann et al 2006). In order to discuss structurally and frequently and evaluate a wide range of problem areas and treatment goals to reach a treatment agreement, SDM intervention was developed.

Numerous modern mental health interventions have supported patient-centered care, patient choice, and selfdirected care, but research on SDM in mental health care is just beginning (Adams and Drake 2006; Joosten et al 2008). To date, only a few studies have explored the effect of SDM on the therapeutic alliance. Results of these studies were contradictory. Bieber et al (2006) found that a computer based information tool for fibromyalgia syndrome patients and SDM communication training for clinicians improved clinician-patient relationship from both clinicians' and patients' perspectives. The effect of this SDM communication training was stronger for patients than for clinicians (Bieber et al 2008). A SDM program for inpatients with schizophrenia appeared to have no effect on clinicians' perception of the therapeutic relationship (Hamann et al 2006). There is extensive literature on related issues, particularly on the therapeutic alliance, but mental health care literature specifically dealing with SDM has just started (Fischer 2006).

We developed a SDM intervention (SDMI) for patients dependent on psychoactive substances in addiction health care programs. Patients and clinicians systematically and frequently discuss and evaluate a wide range of treatment goals. SDMI offers a structured and well-balanced 5-session intervention to reach and carry out joint treatment decisions. The aim of the present study was to examine the effect of a SDMI for substance-dependent patients on the therapeutic alliance. In this study we focused on the therapeutic alliance as an important aspect of the therapeutic relationship. The therapeutic alliance was examined from both patient and clinician perspectives. We hypothesized that patient's and clinician's perceived alliance would improve as a result of SDMI. We also hypothesized that patient's and clinician's perceived alliance will be more congruent when SDMI is carried out.

\section{Methods}

A randomized controlled trial (RCT) was conducted in three addiction treatment centers in the Netherlands. Patients were recruited from January 2005 to May 2006.

\section{Study design}

This study was conducted in clinical practice. Randomization took place at baseline at the level of clinicians and within each site to avoid carry-over effect. We randomized at the level of clinicians to minimize the effect of clinician characteristics on the outcome measure. Elvins and Green (2008) stated that research on therapeutic alliance should focus on RCTs to account for variability in clinician characteristics. Furthermore, this way of randomization was the most feasible and scientifically justifiable procedure. Randomization schedule was generated by computer, and allowed us to randomly allocate the clinicians into two conditions. Clinicians in the experimental condition were trained in the protocols of the SDM intervention method. The other condition consisted of reaching and evaluating a regular treatment agreement (non-structured) without SDMI. Neither study participants nor study staff were blinded to the intervention. Whether or not patients received SDMI depended on the clinician the patients were allocated to. Patients were allocated to one of the clinicians by availability at baseline. Therefore, this allocation can be considered as random because it was only based on accidental events like patients leaving treatment, clinicians' duty, and vacation schedule.

\section{Participants}

Participants were recruited from three addiction treatment centers in the Netherlands. Participating patients were dependent on psychoactive substances and needed inpatient treatment programs. In this study, clinicians were nurses 
or social workers who had most contact with the patients. The clinicians worked at the inpatient treatment unit. All participating centers had comparable 3-month inpatient treatment programs which included individual as well as group aspects. This inpatient treatment included aspects of cognitive behavioral therapy. All patients applying for help in one of these treatment programs during the study period were included in the study. No distinction was made regarding type of substance used. Patients were excluded for the following reasons: being under the age of 18 years; insufficient knowledge of the Dutch language; severe psychiatric co-morbidity that would preclude to take part in the process of SDM and adherence to the protocol; and no informed consent to participate in the study. At follow-up evaluation, patients received a voucher of $€ 20$. The study was approved by the Dutch Ethical Assessment Committee for Experimental Investigations on People (No 4.108).

\section{Measures}

Baseline characteristics were measured by the European Addiction Severity Index (EuropASI) and Composite International Diagnostic Interview - Substance Abuse Module (CIDI-SAM). EuropASI, is a clinical research interview designed to assess problem severity in six areas: physical health; employment; alcohol and/or drug use; legal; family/ social; and psychiatric (McLellan et al 1980). In this study the Dutch version of EuropASI was used which also contains gambling (Hendriks et al 1989; Hartgens et al 1994). Eight severity scores, with ranges 0 (no problem) to 9 (extremely serious problem), were derived from this interview.

Type and severity of substance dependence was assessed by using CIDI-SAM (Compton et al 1996). CIDI-Substance Abuse Module (SAM) is an expanded and more detailed version of the substance use sections of the Composite International Diagnostic Interview (CIDI).

Therapeutic alliance was measured by the Helping Alliance Questionnaire (HAQ). The HAQ is a self-report questionnaire that measures the strength of patient-clinician alliance (Alexander and Luborsky 1984; Luborsky et al 1996). The Dutch version of the HAQ has 11 items which are rated on a 5-point likert scale (completely disagree, disagree, neither disagree nor agree, agree, completely agree) (De Weert-Van Oene et al 1999). A patient, as well as a clinician version, has been developed. This questionnaire measures helping alliance at an individual level (eg, "I feel the therapist understands me."; "I understand the patient."). This HAQ contains 2 scales: Cooperation $(\alpha=0.88)$, a 5 -item scale reflecting the perception of the patient to work together with the therapist and vice versa; and Helpfulness ( $\alpha=0.76$ ), a 5-item scale referring to the patient's confidence in his own capacities in improving the situation and the clinician's confidence in his/her capacities to improve the situation. The helpfulness scale also refers to clinician or treatment effectiveness. HAQ is easy to complete and interpret. Psychometric properties of the HAQ are satisfactory (De Weert-Van Oene et al 1999).

The Goals of Treatment questionnaire was used in the experimental condition as part of the intervention. The Goals of Treatment questionnaire was partly based on the Camberwell Assessment of Need (Phelan et al 1995; McCrone et al 2000; Varo et al 2002) but also covered two additional areas: Gambling and Legal. Areas of problems were translated into "goals" of which the patient could tick whether s/he wanted to work on this definitely, possibly or definitely not. The clinician indicated per goal whether to his/ her opinion the patient had to work on it definitely, possibly or definitely not during treatment.

The procedure of completing the Goals of Treatment questionnaire was extended with a Q-sort ranking (Cronbach 1990) to indicate importance and priority of each goal of this questionnaire. These were cards on which the 24 treatment goals were described. Patient and clinician had to arrange these cards in the same way as the goals of the questionnaire were arranged in (definitely, possibly or definitely not). After this, the piles definitely and possibly were arranged in order of importance and priority. Patients and clinicians could discuss the differences between their indicated priority of each treatment goal by means of these Q-sort cards.

\section{Intervention}

SDMI contains 5 sessions. In the introduction session (session I), at the beginning of the treatment, the clinician introduces the procedure of SDMI to the patient. At the end of this session the patient is handed over the questionnaire and Q-sort cards. One week after the introduction session (session II), patient's treatment goals and expectations are explored and compared with the clinician's perception as described in the results of his questionnaire. Similarities and differences between clinician's and patient's perceptions are discussed. Based on this discussion, the treatment contract is completed. During the interim evaluation (session III), halfway through the treatment, the goals and expectations are explored again with the questionnaire and the results are discussed again and adapted to the treatment development if necessary. At the end of the treatment program, a final evaluation (session IV) takes 
place, based on goals and expectations as put down in the treatment contract. In addition, new goals and expectations are explored on basis of the completed questionnaire and ranked Q-sort cards handed out before this session. In the case of discontinuation of treatment before the interim or final evaluation, if possible, an exit interview with the same content as the final evaluation is carried out. A follow-up evaluation (session $\mathrm{V}$ ) is carried out three months after treatment. In this follow-up meeting the goals and expectations are evaluated which were agreed on during the latest evaluation.

Three months before the start of the study clinicians of the experimental condition were trained in the SDMI protocol and in selected aspects of motivational interviewing (MI) techniques (Rollnick and Miller 1995). In general, MI is used to motivate patients for treatment in addiction health care. So, clinicians in the control condition also used MI. In the experimental condition, MI was offered in a structured way by protocol to explore and compare indicated treatment goals and finally to reach an agreement on these goals. MI represented a small but essential part of SDMI. The 1-day training and a booster session after one month took place per site. A SDMI manual was used in which the sessions were described in detail. Furthermore, clinicians completed a checklist after every SDMI session to check whether they carried out all elements of SDMI.

\section{Procedure}

Data were collected at baseline, interim measurement after 8 weeks of treatment, and end of treatment. At baseline, the first author introduced the study to the patients. After this the patient signed informed consent. Patients' baseline data were collected, such as socio-demographic characteristics, type and severity of substance dependence problems, and DSM-VI diagnosis.

Patients in both SDMI and control condition received a nearly equal amount of 1:1 contact to reach and evaluate the treatment agreement. Most important difference between conditions was the structured and visualized way to reach a treatment agreement in the SDMI condition. In the control condition, decision-making was not as explicit and balanced (who takes the decision) as the SDMI. In the experimental condition, after baseline measurement, the trained clinician introduced SDMI to the patient after which they received SDMI. Patients allocated to control condition received the usual way of reaching a treatment agreement without SDMI. Patient's and clinician's perceived alliance was measured at interim and exit measurement.

\section{Statistical analyses}

Chi-square, or Fisher's exact test for dichotomous data, and independent sample t-test were used to test for differences between SDMI and control condition in relation to baseline characteristics. Baseline analyses were performed for both patients and clinicians who completed at least one measurement of helping alliance.

Analyses were carried out for patients, clinicians, and difference HAQ scores separately. HAQ subscales and total sum scores were computed. Difference scores (patient minus clinician) were computed for each patient-clinician couple for each measuring time-point (eg, patient minus clinician item score at exit). All analyses were conducted for HAQ total, cooperation, and helpfulness score.

The data had a nested structure with patients nested within clinicians. In order to account for the nested data structure in these analyses, Multilevel Modelling (Hox 1998, 2002) was used and estimation was performed using MLwiN (Rasbash et al 2000). Multilevel analysis was performed to explore whether intra-class correlation existed. Two models (without and with clinician variance) were tested regarding the patient HAQ. The difference (deviance dropped from 1363.117 to $1361.535=1.582$ ) had a Chi-squared distribution with 3 degrees of freedom; $p<0.66$; so the added clinician variance did not contribute significantly to the fit. Regarding the clinician HAQ, the difference (deviance dropped from 1326.298 to $1320.268=6.030$ ) had a Chi-squared distribution with 3 degrees of freedom; $p<0.11$; so the added clinician variance did not contribute significantly to the fit. There was no intra-class correlation in this data. Therefore, we were allowed to use independent sample t-tests to compare HAQ scores between SDMI and control condition for each measurement separately.

Finally, Cohen's $d=\mid \mu_{1}-\mu_{2} / \sigma$ was calculated to determine the effect sizes (Cohen, 1988). Cohen defines $d$ 's of $0.2,0.5$, and 0.8 as small, medium, and large effects, respectively.

All statistical tests were two-sided, with a p-value of 0.05 or less considered to indicate statistical significance. All analyses (except multilevel analyses) were performed using SPSS for Windows (release 14.0).

\section{Results}

\section{Patient and clinician characteristics}

Two hundred and sixty one patients were assessed for eligibility. Two hundred and twenty seven patients were approached to participate: 111 patients randomly assigned to SDMI and 116 patients assigned to control condition. Before 
baseline measurement 7 patients refused to provide consent to participate in the study. These 7 patients were assigned to the control condition but were not aware of the condition. Owing to discontinuation of the study at one of the departments, 8 patients did not receive the allocated treatment: 4 in SDMI and 4 in control condition. Two hundred and twelve patients were included in this study: 107 patients were assigned to SDMI and 105 to control condition. We found one significant difference in baseline characteristics between the two condition ( $\mathrm{n}=212)$. Patients' baseline severity score Family/Social Relations was significantly higher among patients who received control intervention $(\mathrm{t}=2.38 ; \mathrm{p}<0.05)$.

Table 1 shows key baseline characteristics of the 147 patients who completed at least one measurement of helping alliance. The remaining 65 patients left treatment before interim measurement or did not complete HAQ at interim or exit measurement. In this reduced sample, only patients' baseline severity score Family/Social Relations was significantly higher among patients who received control intervention $(\mathrm{t}=2.38 ; \mathrm{p}<0.05)$. Clinicians' baseline characteristics are illustrated in Table 2. Professional qualifications of clinicians were nurse or social worker. We found no significant differences between the two conditions regarding clinicians' baseline characteristics.

\section{Helping alliance}

Table 3 illustrates patients' and clinicians' perceived helping alliance by condition at interim and exit measurement.

Table I Baseline characteristics of the study population by condition $(n=147)$

\begin{tabular}{|c|c|c|c|c|c|c|}
\hline \multirow[t]{2}{*}{ Characteristic } & \multirow[t]{2}{*}{ SDMI } & \multicolumn{3}{|c|}{ Control } & \multirow[t]{2}{*}{$\chi^{2}, \mathrm{p}$-value } & \multirow[t]{2}{*}{ t-value, $\mathrm{p}$-value } \\
\hline & & $\mathbf{n}$ & & $\mathbf{n}$ & & \\
\hline Age, mean (SD), years & $40.7(10.3)$ & 64 & $41.2(11.1)$ & 83 & & $0.27,0.79$ \\
\hline Gender, $\%$ male & 76.6 & 49 & 75.9 & 63 & $0.01,1.00$ & \\
\hline Country of birth, $\%$ & & & & & $1.24,0.27$ & \\
\hline Netherlands & 92.2 & 59 & 96.4 & 80 & & \\
\hline Other & 7.8 & 5 & 3.6 & 3 & & \\
\hline Relationship, \% & & & & & $2.68,0.26$ & \\
\hline Married & 12.7 & 8 & 20.7 & 17 & & \\
\hline Divorced/widow & 44.4 & 28 & 32.9 & 27 & & \\
\hline Never married & 42.9 & 27 & 46.3 & 38 & & \\
\hline Employment full or part-time \% & 62.9 & 39 & 53.8 & 42 & I.16, 0.28 & \\
\hline Years of education, mean (SD) & II.3 (2.7) & 64 & $11.6(3.1)$ & 83 & & $0.54,0.59$ \\
\hline \multicolumn{7}{|l|}{ Type of substance dependence, \% } \\
\hline Alcohol dependence & 71.9 & 46 & 74.7 & 62 & $0.15,0.71$ & \\
\hline Drug dependence & 43.8 & 28 & 37.3 & 31 & $0.62,0.50$ & \\
\hline Years of substance use, mean (SD) & $14.8(8.3)$ & 62 & $14.5(10.0)$ & 80 & & $-0.17,0.87$ \\
\hline Primary substance use, $\%$ & & & & & $1.03,0.79$ & \\
\hline Alcohol & 52.4 & 33 & 59.3 & 48 & & \\
\hline Cocaine/Stimulants & 12.7 & 8 & 8.6 & 7 & & \\
\hline Polydrug & 27 & 17 & 25.9 & 21 & & \\
\hline Other & 7.9 & 5 & 6.2 & 5 & & \\
\hline EuropASI severity scores, mean (SD) & & 64 & & 83 & & \\
\hline Physical health & $2.4(2.1)$ & & $2.8(2.1)$ & & & I. $10,0.27$ \\
\hline Work, education, and income & $3.7(1.7)$ & & $3.4(2.0)$ & & & $-1.00,0.32$ \\
\hline Alcohol & $5.2(2.6)$ & & $5.3(2.5)$ & & & $0.34,0.73$ \\
\hline Drugs & $3.4(3.3)$ & & $2.9(3.2)$ & & & $-0.92,0.36$ \\
\hline Legal & $1.5(1.9)$ & & $\mathrm{I} .4(\mathrm{I} .8)$ & & & $-0.12,0.91$ \\
\hline Family/social relations & $3.8(1.5)$ & & $4.4(1.5)$ & & & $2.46,0.02 *$ \\
\hline Psych./emotional problems & $5.8(2.0)$ & & $5.8(2.0)$ & & & $0.03,0.97$ \\
\hline Gambling & $0.5(\mathrm{I} .5)$ & & $0.4(1.2)$ & & & $0.20,0.85$ \\
\hline
\end{tabular}

$*_{p}<0.05$. 
Table 2 Baseline characteristics of the clinicians by condition $(n=34)$

\begin{tabular}{|c|c|c|c|c|c|c|}
\hline \multirow[t]{2}{*}{ Characteristic } & \multirow[t]{2}{*}{ SDMI } & \multicolumn{3}{|c|}{ Control } & \multirow[t]{2}{*}{$\chi^{2}, p$-value } & \multirow[t]{2}{*}{ t-value, $p$-value } \\
\hline & & $\mathbf{n}$ & & $\mathbf{n}$ & & \\
\hline Age, mean (SD), years & $35.6(12.4)$ & 16 & $34.1(12.0)$ & 18 & & $0.36,0.72$ \\
\hline Gender, $\%$ male & 31.3 & 5 & 44.4 & 8 & $0.62,0.50$ & \\
\hline Years of working experience, mean (SD) & |3.| (II.6) & 16 & $12.6(\mid 1.5)$ & 18 & & $0.13,0.90$ \\
\hline
\end{tabular}

Unfortunately, perceived helping alliance was not measured when patients left treatment before interim measurement $(n=78)$. No differences were found between conditions regarding patient's perceived alliance. Patients' scores on the HAQ were particularly high, even at interim measurement (mean scores between 44 and 48 out of a maximum total score of 55).

A significant difference between SDMI and control condition was measured on the HAQ completed by clinicians. Helpfulness $(\mathrm{t}=2.44, \mathrm{p}<0.05$ and $\mathrm{t}=2.82, \mathrm{p}<0.01$ respectively at interim and exit measurement) and total score $(\mathrm{t}=2.01, \mathrm{p}<0.05$ and $\mathrm{t}=3.01, \mathrm{p}<0.01$ respectively at interim and exit measurement) were significantly higher when clinicians carried out SDMI. Table 3 also shows effect sizes calculated by Cohen $d$. Medium effects with ranges $d 0.41$ to 0.62 were found for these significant variables. We also computed a difference score within each patientclinician couple for each assessment time separately (see Table 4). We found a significant difference between conditions concerning HAQ total score at exit measurement $(\mathrm{t}=-2.44$, $\mathrm{p}<0.05)$. The difference between patient and clinician was significantly smaller when SDMI was carried out.

\section{Discussion}

The purpose of this study was to evaluate the influence of a SDMI on patients' and clinicians' perceptions of

Table 3 Patient's and clinician's perceived alliance (HAQ) at interim and exit measurement

\begin{tabular}{|c|c|c|c|c|c|c|c|c|c|}
\hline $\begin{array}{l}\text { HAQ } \\
\text { Mean (SD) }\end{array}$ & $\mathbf{n}$ & SDMI & $\mathbf{n}$ & Control & $\mathbf{n}$ & Total & t-value & P-value & $\begin{array}{l}\text { ES } \\
\text { d }\end{array}$ \\
\hline \multicolumn{10}{|l|}{ Patient } \\
\hline \multicolumn{10}{|l|}{ Interim (T3) } \\
\hline Cooperation & 54 & $20.1(2.5)$ & 66 & 19.7 (3.7) & 120 & $19.9(3.1)$ & 0.73 & 0.46 & 0.13 \\
\hline Helpfulness & 55 & $20.1(2.6)$ & 66 & $20.2(2.8)$ & $12 \mid$ & $20.2(2.7)$ & -0.10 & 0.92 & 0.04 \\
\hline Total & 54 & $44.7(4.1)$ & 66 & 44.I (6.3) & 120 & $44.4(5.4)$ & 0.61 & 0.55 & 0.11 \\
\hline \multicolumn{10}{|l|}{ Patient } \\
\hline \multicolumn{10}{|l|}{ Exit (T4) } \\
\hline Cooperation & 50 & $20.5(2.7)$ & 53 & $21.2(3.2)$ & 103 & $20.8(3.0)$ & -1.13 & 0.26 & 0.24 \\
\hline Helpfulness & 50 & $21.7(2.3)$ & 54 & $21.8(3.0)$ & 104 & $21.8(2.7)$ & -0.14 & 0.89 & 0.04 \\
\hline Total & 50 & $46.6(4.7)$ & 53 & $47.5(6.3)$ & 103 & 47.I (5.6) & -0.82 & 0.42 & 0.16 \\
\hline \multicolumn{10}{|l|}{ Clinician } \\
\hline \multicolumn{10}{|l|}{ Interim (T3) } \\
\hline Cooperation & 52 & $18.9(2.3)$ & 71 & $18.6(2.3)$ & 123 & I8.7 (2.3) & 0.63 & 0.53 & 0.13 \\
\hline Helpfulness & 53 & $19.0(2.4)$ & 73 & $17.8(3.2)$ & 126 & $18.3(3.0)$ & 2.44 & $0.02 *$ & 0.42 \\
\hline Total & 52 & $42.2(4.4)$ & 71 & $40.2(5.4)$ & 123 & $41.0(5.1)$ & 2.01 & $0.05^{*}$ & 0.41 \\
\hline \multicolumn{10}{|l|}{ Clinician } \\
\hline \multicolumn{10}{|l|}{ Exit (T4) } \\
\hline Cooperation & 49 & $18.9(2.3)$ & 47 & I8.3 (2.5) & 96 & $18.6(2.4)$ & 1.10 & 0.27 & 0.25 \\
\hline Helpfulness & 49 & $19.5(2.6)$ & 47 & I7.6 (4.I) & 96 & $18.6(3.5)$ & 2.82 & $0.01 * *$ & 0.55 \\
\hline Total & 48 & $42.6(4.2)$ & 47 & $39.4(6.0)$ & 95 & $4 I .0(5.4)$ & 3.01 & $0.01 * *$ & 0.62 \\
\hline
\end{tabular}


Table 4 Difference score (patient minus clinician) HAQ at interim and exit measurement

\begin{tabular}{|c|c|c|c|c|c|c|c|c|}
\hline $\begin{array}{l}\text { HAQ } \\
\text { Mean (SD) }\end{array}$ & $\mathbf{n}$ & SDMI & $\mathbf{n}$ & Control & $\mathbf{n}$ & Total & t-value & P-value \\
\hline \multicolumn{9}{|l|}{ Interim (T3) } \\
\hline Cooperation & 49 & I.3 (2.8) & 63 & I.I (3.8) & 112 & $1.2(3.4)$ & 0.31 & 0.75 \\
\hline Helpfulness & 50 & I.4 (2.9) & 65 & $2.5(4.4)$ & 115 & $2.0(3.8)$ & -1.66 & 0.10 \\
\hline Total & 49 & $2.9(5.3)$ & 63 & $3.9(8.1)$ & 112 & $3.5(7.0)$ & -0.79 & 0.43 \\
\hline \multicolumn{9}{|l|}{ Exit (T4) } \\
\hline Cooperation & 47 & I.3 (2.9) & 41 & $2.5(3.4)$ & 88 & $1.9(3.2)$ & -1.70 & 0.09 \\
\hline Helpfulness & 48 & $2.1(2.9)$ & 41 & $3.6(4.2)$ & 89 & $2.8(3.7)$ & -1.88 & 0.06 \\
\hline Total & 47 & $3.7(5.4)$ & 41 & $7.0(7.3)$ & 88 & $5.3(6.5)$ & -2.44 & $0.02 *$ \\
\hline
\end{tabular}

$*_{\mathrm{p}}<0.05$.

therapeutic alliance. Results of the present study showed that SDMI resulted in clinicians perceiving the alliance more favorable, and also in greater congruence between clinicians' perceptions and those of the patients. Effect sizes showed a medium effect for these significant findings. There was no significant difference between conditions with regard to patients' perceptions of the alliance. It has to be taken into consideration here that patients in the experimental condition received SDMI on top of an intensive inpatient treatment that was also given to the patients in the control condition.

There are several limitations to the present study that should be noted. Patients and clinicians were not blinded to the intervention and this might have influenced the results. SDMI might have influenced the way of decision-making to reach a treatment agreement within the control condition. Furthermore, therapeutic alliance might have been overestimated by clinicians in the SDMI condition. Results showed that clinicians who carried out SDMI only scored higher on the Helpfulness scale and not on the Cooperation scale. Clinicians should have scored higher on the Cooperation scale if they overestimated the therapeutic alliance. As noted before, HAQ scores were all very high. This ceiling effect is likely to have made it more difficult to demonstrate further improvements in HAQ scores with SDMI. Patient-clinician alliance was measured by a self-report questionnaire and it might be possible that social desirability has influenced the scores. Another limitation is that most patients who left treatment prematurely did not complete the HAQ. It is important to notice that there was no difference between conditions with regard to treatment drop-out. Future research should concern patient's perception of alliance before they drop-out of treatment. Furthermore, it has to be mentioned that patients' baseline severity score on Family/Social Relations was higher among patients who received control intervention. It is not very probable that the higher severity score on Family/Social Relations in the control condition led to a lower score of perceived alliance, although this possibility cannot be excluded. A major strength of this trial is its longitudinal prospective design, with multiple measurement of therapeutic alliance during and after treatment. Other strengths of this study include its multi-centre nature and few barriers to patient recruitment.

Results of this study indicate that SDMI is an effective method to increase clinicians' perceptions of alliance. However, patients' perceptions of the therapeutic alliance were not enhanced by SDMI. This is in contrast with results from a previous study which found that SDM had a greater effect on patients' perceptions than on those of the clinicians (Bieber et al 2008). In this study, fibromyalgia patients received the information tool during the first session before they discussed their treatment plan with their clinician. In our study, substance-dependent patients discussed and evaluated their treatment plan during three SDM sessions. Before these sessions patients and clinicians completed the Goals of Treatment questionnaire to indicate treatment goals and arranged these goals in order of importance and priority by using Q-sort cards.

A reason for not finding differences between patients in SDMI and control condition might be that the HAQ was not sensitive enough to measure greater differences and greater changes over time in the perception of therapeutic alliance due to the ceiling effect. In comparison with patients, clinicians are able to compare the alliance with a specific patient to the alliance with other patients. Perhaps the therapeutic alliance is more a moderating factor of treatment outcome (McGuire et al 2001). More research is therefore needed to explore the effect of SDMI on therapeutic alliance in relation with treatment outcome. 
Clinicians who performed SDMI scored especially higher on the Helpfulness scale. The perception of these clinicians appeared to be more positive regarding their patient's capacities in improving their situation. These clinicians also perceived positive effectiveness of the treatment. This is an important finding because patients diagnosed with substance related disorders are assumed to be more difficult to treat (Najavits et al 2000). SDMI seems to help clinicians to deal with these patients by increasing their perceptions of their own helpfulness. SDMI leads to greater congruence between clinicians' and patients' perceptions of therapeutic alliance. This is not only caused by clinicians' scores but also by patients' scores regarding the perceived alliance. This congruence between the patient and the clinician is favorable for the therapeutic relationship.

Therapeutic alliance is an important topic in addiction treatment. The present study has shown that a specific intervention to enhance shared decision-making leads to a better clinician's perceived alliance and more involvement. This improved alliance is likely to be a helpful prerequisite for good treatment outcome, but further research is needed to demonstrate whether SDMI by improving therapeutic alliance is a significant predictor for improved treatment outcome.

\section{Acknowledgments}

The authors wish to thank staff and patients of the following Dutch Addiction Treatment Centres for their help in completing this project: GGZ Groep Noord- and Midden Limburg, in Venray; Novadic-Kentron, in Breda and Tilburg; and TACTUS, in Rekken and Zutphen. The authors also wish to thank Pierre Souren for his support.

This project is funded by the Dutch Ministry of Welfare and Sports (VWS) and the Dutch Organisation for Health Research and Development (ZonMW) - grant no. 985-10-018. These agencies had no role in the conduct of or interpretation of the study.

Financial support for this study was provided by the Dutch Ministry of Health, Welfare and Sports (VWS) and the Dutch Organization for Health Research and Development (ZonMW). The funding agreement ensured the authors' independence in designing the study, interpreting the data, writing, and publishing the report (grant no. 985-10-018).

\section{Disclosures}

The authors have no conflicts of interest to declare.

\section{References}

Adams JR, Drake RE. 2006. Shared decision-making and evidence-based practice. Community Ment Health J, 42:87-105.

Alexander LB, Luborsky L. 1984. The Penn Helping Alliance Scales. In: Greenberg LS, Pinhof WM, (eds). The psychotherapeutic process:a research handbook. New York: he Guilford Press.

Baldwin SA, Wampold BE, Imel ZE. 2007. Untangling the alliance-outcome correlation:Exploring the relative importance of therapist and patient variability in the alliance. J Consult Clin Psychol, 75:842-52.

Bieber C, Müller KG, Blumenstiel K, et al. 2006. Long-term effects of a shared decision-making intervention on physician-patient interaction and outcome in fibromyalgia. A qualitative and quantitative 1 year follow-up of a randomized controlled trial. Patient Educ Couns, 63:357-66.

Bieber C, Müller KG, Blumenstiel K, et al. 2008. A shared decision-making communication training program for physicians treating fibromyalgia patients: ffects of a randomized controlled trial. J Psychosom Res, 64:13-20.

Charles C, Whelan T, Gafni A. 1999. What do we mean by partnership in making decisions about treatment? BMJ, 319:780-2.

Cohen J. 1988. Statistical power analysis for the behavioural sciences (2nd edition). Hillsdale NJ:Lawrence Earlbaum Associates.

Compton WM, Cottler LB, Dorsey KB, et al. 1996. Comparing assessments of DSM-IV substance dependence disorders using CIDI-SAM and SCAN. Drug Alcohol Depend, 41:179-87.

Connors GJ, Carroll KM, DiClemente CC, et al. 1997. The therapeutic alliance and its relationship to alcoholism treatment participation and outcome. J Consult Clin Psychol, 65:588-98

Crawford MJ, Aldridge T, Bhui K, et al. 2003. User involvement in the planning and delivery of mental health services: a cross-sectional survey of service users and providers. Acta Psychiatr Scand, 107:410-4.

Cronbach LJ. 1990. Essentials of Psychological Testing. New York: Harper and Row.

De Jong-Verhagen JG, De Jong CAJ. 2001. Cliënten en behandelingsteam: op een lijn? Een methode voor het inschatten van doelen en verwachtingen van cliënten en behandelingsteam in een beschermende woonvoorziening. (Clients and treatment staff:at the same level? A method to estimate treatment goals and expectations of clients and treatment staff in supported housing). $M G v, 56: 1057-71$.

De Weert-Van Oene GH, De Jong CAJ, Jorg F, et al. 1999. The helping Alliance Questionnaire:Psychometric Properties in Patients with substance dependence. Subst Use Misuse, 34:1549-69.

Elvins R, Green J. 2008. The conceptualization and measurement of therapeutic alliance:An empirical review. Clin Psychol Review, doi:10.1016/ j.cpr.2008.04.002.

Fischer EP. 2006. Shared decision making and evidence-based practice: A commentary. Community Ment Health J, 42:107-11.

Fuertes JN, Mislowack A, Bennett J, et al. 2007. The physician-patient working alliance. Patient Educ Couns, 66:29-36.

Greenson RR. 1965. The working alliance and the transference neuroses. Psycho-analysis Quarterly, 34:155-81.

Hamann J, Leucht S, Kissling W. 2006. Shared decision making for in-patients with schizophrenia. Acta Psychiatr Scand, 114:265-73.

Hartgens C, Hendriks V, Van der Meer CW, et al. 1994. De Nederlandse vertaling van de Europese versie van de Addiction Severity Index, $5^{\mathrm{e}}$ editie (Dutch Translation of the European Version of the Addiction Severity Index, 5th ed). Amsterdam:AIAR.

Hendriks VM, Kaplan CD, Van Limbeek J, et al. 1989. The Addiction Severity Index:reliability and validity in a Dutch addict population. J Subst Abuse Treat, 6:133-41.

Hougaard E. 1994. The therapeutic alliance - A conceptual analysis. Scan J Psychol, 35:67-85.

Hox J. 1998. Multilevel modelling:when and why? In Balderjahn I, Mathar $\mathrm{R}$, Schader M. eds. Classification, data analysis, and data highway. New York: Springer Verlag.

Hox J. 2002. Multilevel analysis. Techniques and applications. New Jersey: Lawrence Erlbaum Associations, Inc. 
Joosten EAG, DeFuentes-Merillas L, De Weert GH, et al. 2008. Systematic review of the effects of shared decision making on patient satisfaction, treatment adherence and health status. Psychother Psychosom, 77:219-26.

Luborsky L, Barber J, Siqueland L, et al. 1996. The revised Helping Alliance Questionnaire (HAq-II). J Psychother Pract Res, 5:260-71.

Luborsky L. 1976. Helping alliances in psychotherapy. The groundwork for a study of their relationship to its outcome. In Cleghorn JL, ed. Successful Psychotherapy. New York: Brunner/Mazel. pp. 92-116.

McCrone P, Leese M, Thornicroft G, et al. 2000. Reliability of the Camberwell Assessment of Need- European Version. Epsilon study 6. European psychiatric services:inputs linked to outcome domains and needs. Br J Psychiatry, 39; 34-40.

McGuire R, McCabe R, Priebe S. 2001. Theoretical frameworks for understanding and investigating the therapeutic relationship in psychiatry. Soc Psychiatry Psychiatr Epidemiol, 36:557-64.

McLellan AT, Luborsky L, Woody GE, et al. 1980. An improved diagnostic evaluation instrument for substance abuse patients:the addiction severity index. J Nerv Ment Dis, 168:26-33.

Meier PS, Barrowclough C, Donmall MC. 2005. The role of the therapeutic alliance in the treatment of substance misuse:a critical review of the literature. Addiction, 100:304-16.
Najavits LM, Crits-Christoph P, Dierberger A. 2000. Clinicians' impact on the quality of substance use disorder treatment. Subst Use Misuse, 35:2161-90.

Phelan M, Slade M, Thornicroft G, et al. 1995. The camberwell assessment of need:the validity and reliability of an instrument to assess the needs of people with severe mental illness. Br J Psychiatry, 167:589-95.

Rasbash J, Browne W, Goldstein H, et al. 2000. A user's guide to MlwiN (2nd edition). London:Institute of Education.

Robinson A, Thomson R. 2001. Variability in patient preferences for participating in medial decision making:implication for the use of decision support tools. Qual Health Care, 10(Suppl):34-8.

Rollnick S, Miller WR. 1995. What is motivational interviewing? Behavioural and Cognitive Psychotherapy, 23:325-34.

Sanchez-Craig M. 1990. Brief didactic treatment for alcohol and drugsrelated problems:an approach based on client choice. Addiction, 85:169-77.

Van der Weijden T, Van Veenendaal H, Timmermans D. 2007. Shared decision making in the Netherlands - current state and future perspectives. Z Arztl Fortbild Qualitätssich, 101:241-6.

Varo CR, Torres Gonzalez F, Luna del Castillo J, et al. 2002. Reliability of the Spanish version of the camberwell assessment of needs. Actas Esp Psiquiatr, 30:99-104. 
